\title{
Possible Role of P-Glycoprotein in Cyclosporine - Acitretin Drug Interaction
}

\author{
Subhashri SHR and Vimalavathini R*
}

\begin{abstract}
P-glycoprotein, an efflux transporter prevents intracellular accumulation of xenobiotics. Cyclosporine $A$ and acitretin are used to treat psoriasis. The present research work aims to investigate the possible role of $P$-glycoprotein in Cyclosporine- Acitretin drug interaction due to evidence of both drugs having affinity for P-glycoprotein. The study has three different objective namely to assess apparent permeability coefficient of cyclosporine in presence of acitretin by non-everted sac technique, to study the intestinal permeation and absorption kinetics of cyclosporine in presence of acitretin by single pass intestinal perfusion (SPIP) technique and to study the influence of acitretin on oral bioavailability of cyclosporine in Wistar rats. The result of ex vivo, in situ and in vivo study showed that cyclosporine level increased in a time dependent manner. As the dose of acitretin increases the cyclosporine concentration in all three methods tend to increase and was statistically significant. The improvement in absorption of cyclosporine may be due to the inhibition of $P$ glycoprotein transporter in the intestine by acitretin. Thus acitretin may enhance the oral pharmacokinetics of cyclosporine. Hence this combination may require close monitoring for better therapeutic outcome. Our study confirmed the inhibitory role of acitretin on P-glycoprotein leading to increase concentration of cyclosporine.
\end{abstract}

Index Terms-Acitretin, Cyclosporine, drug interaction, Pglycoprotein.

\section{INTRODUCTION}

Drug transporters and metabolizing enzymes play vital role in determining the pharmacological action of drugs. Pglycoprotein (P-gp) is an active, efflux, membrane bound transport protein pump encoded by multi-drug resistance (MDR1) gene, also known as ABCB1 gene in humans [1]. It is expressed in the apical membranes of intestinal epithelial cells, biliary canalicular membranes, renal proximal tubular epithelial cells, capillary endothelial cells of the bloodbrain-barrier, it is an efflux pump to decrease the intracellular accumulation of xenobiotic [2]. P-gp due to genetic polymorphism may affect drug therapy in two ways first, it increases the expression of P-gp thereby it reduces the bioavailability and leads to therapeutic failure and secondly, it decreases P-gp expression and thereby produces drug toxicity [3]. It is highly expressed in cancer cells, leading to efflux of anticancer agents from cells which confer chemotherapeutic drug resistance [4].

Published on September 6, 2019.

Subhashri SHR is with Department of Pharmacology, College of Pharmacy, Mother Theresa Post Graduate and Research Institute of Health sciences, Puducherry, India (e-mail: subhasnow@gmail.com).

Vimalavathini R, Assistant Professor is with Department of Pharmacology, College of Pharmacy, Mother Theresa Post Graduate and Research Institute of Health sciences, Puducherry, India (e-mail: vimalavathini@gmail.com).
Psoriasis is a disease which requires quick response to therapy. Based of different mechanism of action and different side effect profilecombined treatment of cyclosporine $\mathrm{A}$ and acitretin are used to treat psoriasis. One study showed there were a few clinical cases that do not respond well to combination therapy of these two drugs [5]. Cyclosporine is a substrate of P-gp and in silico and in vitro cell culture studies show that acitretin also has affinity for Pgp [6]. Thus we hypothesize possible drug interaction between these two drugs mediated by P-gp which may affect the effectiveness of this combination therapy. The aim of the present study was to investigate the possible role of P-gp in cyclosporine-acitretin drug interaction.

The study has three different objective namely to assess apparent permeability coefficient of cyclosporine in presence of acitretin using Wistar rats by non-everted sac technique, to study the intestinal permeation and absorption kinetics of cyclosporine in presence of acitretin using Wistar rats by single pass intestinal perfusion (SPIP) study and to study the influence of acitretin on oral bioavailability of cyclosporine in Wistar rat.

\section{MATERIALS AND METHODS}

Cyclosporine A (Panacea Biotec Ltd., India) and acitretin (A-TRET, Kaizen Research Labs India Pvt Ltd.). Rat cyclosporine A (CsA) Elisa Kit (Shanghai Korain Biotech Co.Ltd., China) and LDH and Glucose kit (Randox Laboratories Pvt Ltd., India) were purchased.

Albino Wistar rats weighing 150-200g of either sex were procured from Biogen Laboratory Animal Facility, Bangalore. The animals were housed in clean polypropylene cages in an air conditioned room and were kept under standard conditions of humidity $(50 \pm 5) \%$, temperature $\left(25 \pm 2^{\circ} \mathrm{C}\right)$ and light $(12 \mathrm{~h} \mathrm{light} / 12 \mathrm{~h}$ dark cycle). Rats were fed with standard pellet diet and water provided ad libitum. They were initially acclimatized to the laboratory environment for seven days prior to their use. The study protocol is approved (CPCSEA / 1923 / Re / AHF / MTPG $\&$ RIHS / $2^{\text {nd }}$ IAEC 2018/03) by the Institutional Animal Ethics Committee.

\section{I. $\quad$ Non-Everted Sac technique}

Drug treatment consists of three different groups Group 1 cyclosporine $(30 \mu \mathrm{M})$, Group 2 cyclosporine $(30 \mu \mathrm{M})+$ Acitretin $(100 \mu \mathrm{M})$, Group 3 received cyclosporine $(30 \mu \mathrm{M})$ + Acitretin $(150 \mu \mathrm{M})$. Wistar rats $(\mathrm{n}=2)$ were fasted overnight with free access to water before the experiment. They were sacrificed with overdose of pentobarbitone 
sodium $(50 \mathrm{mg} / \mathrm{kg} / \mathrm{i} . \mathrm{p})$. The small intestine was removed and flushed with $50 \mathrm{~mL}$ ice-cold saline. The jejunum were isolated, and a segment was cut into three different lengths of $10 \mathrm{~cm}$ for preparation of the sac. Tyrode's solution containing drug were introduced into the non-everted sac (mucosal side), and both ends of the sac were ligated tightly. The sac was mounted in $40 \mathrm{ml}$ organ bath with Tyrode solution oxygenated with $5 \% \mathrm{CO}_{2} / 95 \% \mathrm{O}_{2}$ at $37{ }^{\circ} \mathrm{C}$. Drug sampling was done at 30,60, 90, 120 mins and replenished with fresh buffer [7] was stored at $-20^{\circ} \mathrm{C}$ and cyclosporine was determined using Rat cyclosporine A (CsA) Elisa Kit. The LDH of non everted sac was measured at 30, 60, 90, and 120 mins and compared to the cyclosporine treated group. The concentration of cyclosporine was measured by using Rat cyclosporine A (CsA) Elisa Kit. LDH, glucose transport was measured using commercial available kits.

\section{Apparent permeability coefficient $\left(P_{a p p}\right)$}

The apparent permeability coefficient for cyclosporine was calculated from the equation (1) given below.

$$
P_{a p p}=\frac{d Q}{d t} \cdot \frac{1}{A C O}
$$

Where,

$\mathrm{dQ} / \mathrm{dt}$ - The rate of drug transport( cyclosporine) from mucosal to serosal medium

A- The surface area of the intestinal sac used for the study $\left(\mathrm{cm}^{2}\right)$

CO- The initial concentration of drug present in the intestinal sac

\section{II. $\quad$ Single Pass intestinal perfusion (SPIP)}

Drug treatment consists of three different groups Group 1 received cyclosporine $(50 \mu \mathrm{M})$, Group 2 received cyclosporine $(50 \mu \mathrm{M})+$ Acitretin $(100 \mu \mathrm{M})$, Group 3 received cyclosporine $(50 \mu \mathrm{M})+$ Acitretin $(200 \mu \mathrm{M})$. Adult Wistar rats $(n=4)$ were fasted overnight with free access to water, anesthetized using an intraperitoneal injection of pentobarbitone sodium $(50 \mathrm{mg} / \mathrm{kg})$ and placed on a heated pad to maintain normal body temperature. Upon verification of the loss of pain reflex, a midline longitudinal abdominal incision was made, and the lumen of the jejunum $(10 \mathrm{~cm})$ was flushed with $10 \mathrm{ml}$ of saline pre-warmed to $37^{\circ} \mathrm{C}$.The proximal end of the lumen was catheterized with an inlet polypropylene tube for inflow of buffer containing drug solution at flow rate of $0.2 \mathrm{ml} / \mathrm{min}$. The distal end of the jejunum was also catheterized with an outlet polypropylene tube to collect intestinal effluent. The entire excised area was covered with an absorbable cotton pad soaked in normal saline to prevent dryness of intestine. After allowing 30 mins to reach steady-state outlet concentration, outlet perfusate samples were collected at every $15 \mathrm{mins}$ time interval for a period of 120 mins. At the end, the length of the segment was measured without stretching and intestinal weight also weighed [8], [9]. The radius of the intestine was taken to be $0.18 \mathrm{~cm}$. Finally, the animal was euthanatized. Cyclosporine was determined using Rat cyclosporine A (CsA) Elisa Kit.

\section{Effective permeability coefficient $\left(P_{\text {eff }}\right)$}

The effective permeability coefficient of the drug was calculated from the following equation (2).

$$
P_{\text {eff }}=\frac{-Q_{\text {in }} * \ln \left(\frac{\operatorname{Cout}_{(\text {corr })}}{\operatorname{Cin}}\right)}{2 \pi r L}
$$

Where;

Peff-Effective Permeability Coefficient $(\mathrm{Cm} / \mathrm{Sec})$

$Q_{\text {in-Perfusion flow rate }(0.2 \mathrm{ml} / \mathrm{min})}$

$\mathrm{C}_{\text {out}}$-Corrected outlet drug concentration

$\mathrm{C}_{\mathrm{in}}$-Corrected inlet drug concentration

R-Radius of the rat small intestine $(0.18 \mathrm{Cm})$

L-Length of the perfused intestinal segment $(\mathrm{Cm})$

\section{Absorption rate constant (ka)}

Absorption rate constant $\mathrm{ka}$ and $\mathrm{C}_{\text {out(corr) }}$ were calculated from the following equation (3).

$$
\begin{aligned}
& K a=\frac{\left(1-\frac{\operatorname{Cout}_{(\text {corr })}}{\operatorname{Cin}}\right) * Q_{\text {in }}}{V} \\
& \operatorname{Cout}_{(\text {Corr })}=\operatorname{Cout} * \frac{Q_{\text {out }}}{Q_{\text {in }}} \\
& V=\pi R^{2} L
\end{aligned}
$$

Where, $\mathrm{C}_{\text {out(corr) }}$ is the water flux corrected concentration of the compound measured in the exiting perfusate at the specified time interval $(15,30,45,60,75,90,105,120$ minutes); $\mathrm{C}_{\mathrm{in}}$ denotes drug concentration of the drug measured in entering perfusate; $Q_{\text {in }}$ is the perfusion rate $(0.2$ $\mathrm{mL} / \mathrm{min}$ ); and $\mathrm{V}$ is the volume of perfused segment.

The net water flux (NWF) in the in situ perfusion studies (water absorption and efflux in the intestinal segment) was determined by gravimetric method. Net water flux was calculated by below equation (4);

$$
N W F=\left[1-\left(\frac{Q_{\text {out }}}{Q_{\text {in }}}\right)\right] * Q_{\text {in }} / l
$$

Where;

$Q_{i n}$ is the measured flow rate $(\mathrm{m} \mathrm{L} / \mathrm{min})$ of entering intestinal perfusate

$Q_{\text {out }}$ is the measured flow rate (m $\mathrm{L} / \mathrm{min}$ ) of exiting intestinal perfusate for specified time interval calculated from the actual intestinal perfusate density $(\mathrm{g} / \mathrm{m} \mathrm{L})$

$l$ is the length $(\mathrm{cm})$ of intestinal segment perfusate

The human values and fraction of drug absorption were predicted from previous study by using the following equation(5);

$$
\begin{aligned}
& P_{\text {eff (human })}=1.04 P_{\text {eff }(\text { rat })}-0.0003 * 10^{-4} \\
& F_{a(\text { human })}=1-e^{-2 * P_{\text {eff }(\text { rat })}}
\end{aligned}
$$

\section{Effect of acitretin on oral bioavailability of} cyclosporine 
Adult Wistar rats $(\mathrm{n}=4)$ were fasted for 24 hours. Drug treatment consists of three different groups Group 1 received Cyclosporine $(1 \mathrm{mg} / \mathrm{kg} / \mathrm{p} . \mathrm{o})$, Group 2 received cyclosporine $(1 \mathrm{mg} / \mathrm{kg} / \mathrm{p} . \mathrm{o})+$ Acitretin $(5 \mathrm{mg} / \mathrm{kg} / \mathrm{p} . \mathrm{o})$, Group 3 received Cyclosporine (1mg/kg/p.o) + Acitretin (10mg/kg/p.o) [10]. After that each group's was treated with cyclosporine and 1 hour later vehicle or acitretin was administrated per orally and blood sample were withdrawn by retro-orbital plexus 2 hours after acitretin administration [11]. Concentration of cyclosporine in blood was estimated using Rat cyclosporine A (CsA) Elisa Kit.

\section{STATISTICAL ANALYSIS}

Statistical analysis was carried out using SPSS version 16.0 (SPSS Inc., Chicago, IL, USA). All parameter was represented as mean \pm SEM. One-way ANOVA followed by Bonferroni's multiple comparison tests was used to compare groups. A value of $\mathrm{p}<0.05$ was considered to be statistically significant.

\section{RESULTS}

\section{A. Ex-vivo: Non- everted sac technique}

TABLE I: Apparent Permeability Coefficient $\left(\mathrm{P}_{\mathrm{app}}\right)$ of cyclosporine in presence or absence of acitretin in rats using noneverted sac technique

\begin{tabular}{|l|c|c|c|}
\hline Drug treatment & \multicolumn{3}{|c|}{ Apparent permeability (P ) Cm/s } \\
\cline { 2 - 4 } & 30mins & 60mins & 90mins \\
\hline Cyclosporine $(30 \mu \mathrm{M})$ & $0.069 \pm 0.003$ & $0.153 \pm 0.001$ & $0.22 \pm 0.002$ \\
\hline Cyclosporine $(30 \mu \mathrm{M})+$ Acitretin $(100 \mu \mathrm{M})$ & $0.07 \pm 0.002 *$ & $0.196 \pm 0.002 *$ & $0.292 \pm 0.003 *$ \\
\hline Cyclosporine $(30 \mu \mathrm{M})+$ Acitretin $(150 \mu \mathrm{M})$ & $0.072 \pm 0.001 * *$ & $0.233 \pm 0.002 * *$ & $0.345 \pm 0.003 * *$ \\
& & $0.513 \pm 0.003 * *$ \\
\hline
\end{tabular}

Data expressed as mean \pm SEM ( $n=2)$. One-way ANOVA followed by Bonferroni's multiple comparison test. A value of $* \mathrm{p}<0.05, * * \mathrm{p}<0.01$ when compared cyclosporine alone treated group.

There was an increase in the apparent permeability coefficient for cyclosporine in a time dependent manner. Also the apparent permeability coefficient for groups treated with cyclosporine and acitretin at low $(0.31 \pm 0.002$ vs $0.433 \pm 0.002 ; \quad \mathrm{p}<0.05)$ and high dose $(0.31 \pm 0.002$ vs $0.513 \pm 0.003 ; \mathrm{p}<0.01$ ) increased when (table 1) compared to cyclosporine alone treated group and was statistically significant.

Fig. 1: LDH release in cyclosporine in absence or presence of acitretin treated groups in rat non-everted sac model.

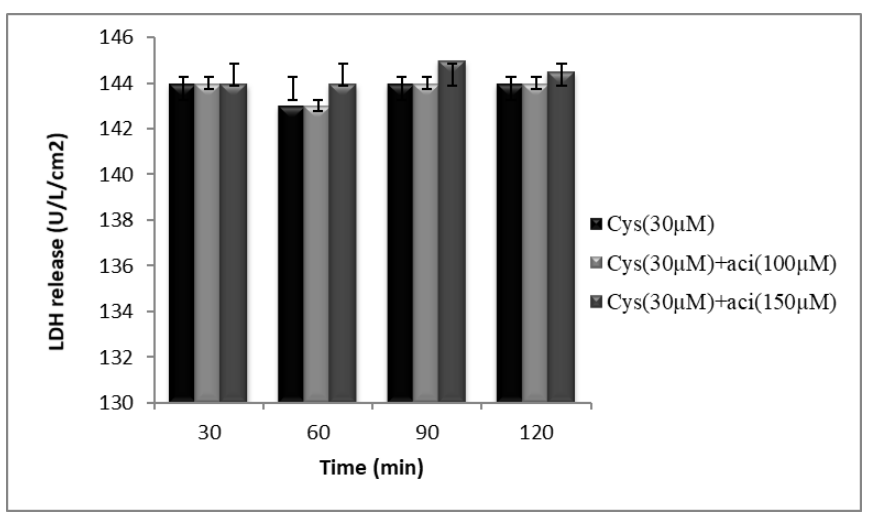

Data expressed as mean $\pm \operatorname{SEM}(n=2)$. and acitretin at low dose, cyclosporine and acitretin at high dose treated groups in rat non-everted gut sac model



Data expressed as mean $\pm \operatorname{SEM}(n=2)$.

There was no significant difference in LDH activity in all the three groups till $120 \mathrm{~min}$ (fig. 1). There was no significant difference in glucose concentration ratio i.e concentration of glucose inside the sacs (serosal side) was and outside (mucosal) concentration for all three (fig. 2) groups at various time interval.

\section{B. In situ: single pass intestinal perfusion technique}

Fig. 2: Glucose transport in cyclosporine, cyclosporine

Table II: Effective permeability coefficient $\left(\mathrm{P}_{\mathrm{eff}}\right)$ of cyclosporine with or without acitretin in rats using single pass intestinal perfusion technique. 


\begin{tabular}{|c|c|c|c|c|}
\hline \multicolumn{2}{|c|}{ Drug treatment } & \multirow{2}{*}{$\begin{array}{c}\text { Cyclosporine }(50 \mu \mathrm{M}) \\
0.0088 \pm 0.0002\end{array}$} & \multirow{2}{*}{$\begin{array}{c}\text { Cyclosporine }(50 \mu M) \\
+ \text { Acitretin }(\mathbf{1 0 0} \mu \mathrm{M})\end{array}$} & \multirow{2}{*}{$\begin{array}{c}\begin{array}{c}\text { Cyclosporine }(50 \mu \mathrm{M})+ \\
\text { Acitretin }(200 \mu \mathrm{M})\end{array} \\
0.0093 \pm 0.0004^{* *}\end{array}$} \\
\hline \multirow{8}{*}{$\begin{array}{l}\text { Effective permeability } \\
\left(P_{\text {eff }}\right) \mathrm{cm} / \mathrm{s}\end{array}$} & 15 mins & & & \\
\hline & 30 mins & $0.0126 \pm 0.0005$ & $0.0152 \pm 0.00012 *$ & $0.0173 \pm 0.00013 * *$ \\
\hline & 45 mins & $0.0165 \pm 0.0001$ & $0.0186 \pm 0.00011^{*}$ & $0.021 \pm 0.0001 * *$ \\
\hline & 60 mins & $0.0198 \pm 0.0001$ & $0.0215 \pm 0.0001 *$ & $0.023 \pm 0.0001 * *$ \\
\hline & 75 mins & $0.022 \pm 0.0001$ & $0.023 \pm 0.0005^{*}$ & $0.0243 \pm .0 .0001 * *$ \\
\hline & 90 mins & $0.023 \pm 0.0001$ & $0.024 \pm 0.007 *$ & $0.0254 \pm 0.0005 * *$ \\
\hline & 105 mins & $0.0239 \pm 0.0004$ & $0.0254 \pm 0.0001 *$ & $0.0267 \pm 0.0001 * *$ \\
\hline & 120 mins & $0.0242 \pm 0.0001$ & $0.0264 \pm 0.0002 *$ & $0.0283 \pm 0.0001 * *$ \\
\hline
\end{tabular}

Data expressed as mean \pm SEM $(n=4)$. One-way ANOVA followed by Bonferroni's multiple comparison tests. A value of $* \mathrm{p}<0.05, * * \mathrm{p}<0.01$ when compared cyclosporine alone treated group.

There was an increase in the effective permeability coefficient for cyclosporine in a time dependent manner. Effective permeability coefficient for groups treated with cyclosporine and acitretin at low $(0.0242 \pm 0.0001$ vs $0.0264 \pm 0.0002)$ and high dose $(0.0242 \pm 0.0001$ vs
$0.0283 \pm 0.0001$ ) increased (Table II) when compared to cyclosporine alone treated group and was statistically significant.

Table III: Absorption rate constant $(\mathrm{Ka})$ of cyclosporine with or without acitretin in rats single pass intestinal perfusion technique.

\begin{tabular}{|c|c|c|c|c|}
\hline \multicolumn{2}{|c|}{ Drug treatment } & Cyclosporine $(50 \mu \mathrm{M})$ & Cyclosporine $(50 \mu M)+$ & Cyclosporine $(50 \mu M)+$ \\
\hline \multirow{8}{*}{$\begin{array}{c}\text { Absorption rate } \\
\text { constant(Ka) } \\
\left(\text { Mins }^{-1}\right)\end{array}$} & 15mins & $0.4 \pm 0.019$ & $0.43 \pm 0.024 *$ & $0.45 \pm 0.071^{* *}$ \\
\hline & 30mins & $0.83 \pm 0.03$ & $1.22 \pm 0.026^{*}$ & $1.7 \pm 0.042 * *$ \\
\hline & 45mins & $1.5 \pm 0.017$ & $2.05 \pm 0.023^{*}$ & $2.6 \pm 0.029 * *$ \\
\hline & 60mins & $2.4 \pm 0.084$ & $3.1 \pm 0.033^{*}$ & $3.6 \pm 0.031 * *$ \\
\hline & 75mins & $3.3 \pm 0.045$ & $3.68 \pm 0.025^{*}$ & $4.2 \pm 0.014^{* * *}$ \\
\hline & 90mins & $4 \pm 0.076$ & $4.5 \pm 0.028 *$ & $5.14 \pm 0036 * *$ \\
\hline & $105 \mathrm{mins}$ & $4.17 \pm 0.068$ & $5.25 \pm 0.028^{*}$ & $6.02 \pm 0.046^{* *}$ \\
\hline & $120 \mathrm{mins}$ & $4.4 \pm 0.061$ & $5.9 \pm 0.073 * *$ & $7.7 \pm 0.005^{* *}$ \\
\hline
\end{tabular}

Data expressed as mean \pm SEM ( $\mathrm{n}=4)$. One-way ANOVA followed by Bonferroni's multiple comparison test. A value of ${ }^{*} \mathrm{p}<0.05,{ }^{*} \mathrm{p}<0.01$ when compared cyclosporine alone treated group.

There was an increase in the absorption rate constant for cyclosporine in a time dependent manner. Absorption rate constant for groups treated with cyclosporine and acitretin at low (4.4 \pm 0.061 vs $5.9 \pm 0.073)$ and high dose $(4.4 \pm 0.061$ vs
$7.7 \pm 0.005)$ increased when compared to cyclosporine alone treated group and (Table III) was statistically significant.

Table IV: Predicted Human $P_{\text {eff }}$ values of cyclosporine in rats using single pass intestinal perfusion technique

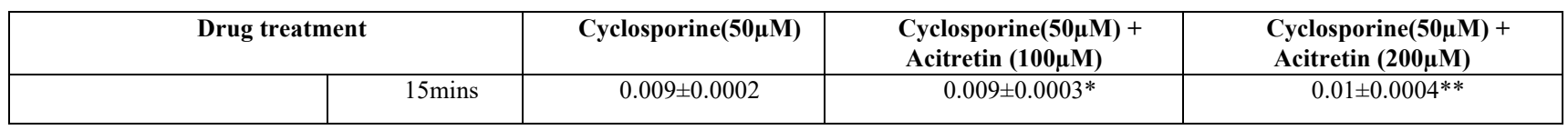




\begin{tabular}{|c|c|c|c|c|}
\hline & $30 \mathrm{mins}$ & $0.0137 \pm 0.0004$ & $0.0157 \pm 0.0001^{*}$ & $0.0179 \pm 0.0001^{* *}$ \\
\cline { 2 - 5 } (Human P eff $\left._{\mathbf{4}} \mathbf{c m} / \mathbf{s}\right)$ & $45 \mathrm{mins}$ & $0.0171 \pm 0.0001$ & $0.0193 \pm 0.0001^{*}$ & $0.021 \pm 0.0001^{* *}$ \\
\cline { 2 - 5 } & $60 \mathrm{mins}$ & $0.02 \pm 0.0001$ & $0.0213 \pm 0.0001^{*}$ & $0.023 \pm 0.0001^{* *}$ \\
\cline { 2 - 5 } & $75 \mathrm{mins}$ & $0.022 \pm 0.0001$ & $0.0232 \pm 0.0001^{*}$ & $0.025 \pm 0.0001^{* *}$ \\
\cline { 2 - 5 } & $90 \mathrm{mins}$ & $0.024 \pm 0.0001$ & $0.0249 \pm 0.0001^{*}$ & $0.0263 \pm 0.0001^{* *}$ \\
\cline { 2 - 5 } & $105 \mathrm{mins}$ & $0.0248 \pm 0.0003$ & $0.0264 \pm 0.0001^{*}$ & $0.0277 \pm 0.0001^{* *}$ \\
\cline { 2 - 5 } & $120 \mathrm{mins}$ & $0.025 \pm 0.0001$ & $0.0274 \pm 0.0001^{* *}$ & $0.0293 \pm 0.0001^{* *}$ \\
\hline
\end{tabular}

Data expressed as mean \pm SEM $(n=4)$. One-way ANOVA followed by Bonferroni's comparison test. A value of * $p<0.05$, $* * \mathrm{p}<0.01$ when compared cyclosporine alone treated group.

The predicted humans' value $\left(\mathrm{P}_{\mathrm{eff}}\right.$ (human) $)$ shows that there was an increase in the cyclosporine concentration in a time dependent manner. Predicted human values for groups treated with cyclosporine and acitretin at low $(0.025 \pm 0.0001$ vs $0.0274 \pm 0.0001)$ and high dose $(0.025 \pm 0.0001$ vs $0.0293 \pm 0.0001)$ increased when compared to cyclosporine alone treated group and was statistically significant (Table IV). The fraction of drug absorption for each group were predicted and found to be $53 \%, 60 \%$, and $65 \%$ respectively.

Fig.3: Effect of acitretin on plasma concentration of cyclosporine in presence or absence of acitretin in Wistar rats

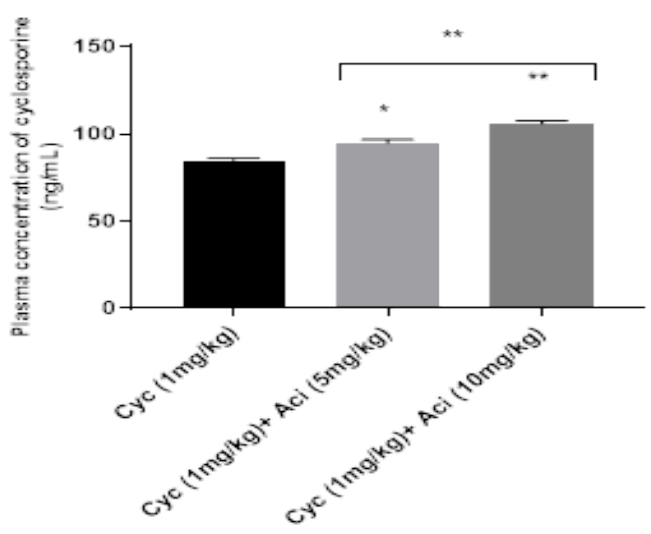

Data expressed as mean \pm SEM $(n=4)$. One-way ANOVA followed by Bonferroni's multiple comparison test. A value of $* \mathrm{p}<0.05, * * \mathrm{p}<0.01$ when compared cyclosporine alone treated group.

Overall in each group the mean plasma concentration of cyclosporine increased in rats treated with cyclosporine and acitretin at low $(84.75 \pm 1.49 \mathrm{vs} 94.75 \pm 2.01 ; \mathrm{p}<0.05)$ and high dose $(84.75 \pm 1.49 \mathrm{vs} 106 \pm 1.58 ; \mathrm{p}<0.01)$ and (fig. 3$)$ were statistically significant.

\section{Discussion}

Ex vivo study result reveals that in apparent permeability coefficient $\left(\mathrm{P}_{\mathrm{app}}\right)$ of cyclosporine increased in presence of acitretin in a dose dependent manner. LDH levels in ex vivo revealed no significance till $120 \mathrm{~min}$, thus indicating there was no cell damage during experimental period. In each groups concentration of glucose inside the sacs (serosal side) was approximately 1.2 times higher than the outside (mucosal) concentration, indicating that the tissue of sac was viable and well-functioning. Glucose is actively transported in the small intestine, and a well maintained glucose gradient between the external medium and serosal fluid indicates intact and metabolically active tissue [12].

In situ study the effective permeability coefficient, absorption rate constant, predicted humans $P_{\text {eff }}$ value exhibited an increase in cyclosporine level in a dose dependent manner. Gravimetric method analysis showed that there were no significant changes in the in situ inlet concentration with time. The membrane integrity thus has been maintained during perfusion study.

In vivo study plasma concentration of cyclosporine significantly increased when they were co-administered with acitretin indicating that acitretin enhanced the oral bioavailability of cyclosporine. All three methods exhibited that cyclosporine and acitretin at low dose, cyclosporine and acitretin at high dose treated groups increased in time dependent and dose dependent manner.

Both acitretin and cyclosporine $\mathrm{A}$ are metabolized by cyctochrome P450 enzyme. Kuijpers et al showed that cyclosporine A metabolism was inhibited by $33-45 \%$ by etretinate, a metabolite of acitretin. Thus the improvement in absorption of cyclosporine may be due to the inhibition of P-gp and CYP3A4 in the intestine and liver by acitretin. Hence our study shows that enhancement of cyclosporine absorption may be not only due to enzyme inhibition alone but also P-gp inhibition, suggesting that combined use of acitretin and cyclosporine may be useful in reducing the dose of cyclosporine and also may require close monitoring of potential drug interactions. This drug interaction may be clinically significant because cyclosporine is a drug with severe adverse effects even at therapeutic doses and hence slightly increase in plasma concentration of cyclosporine may causes detrimental consequences on therapeutic use.

\section{CONCLUSION}

Concentration of cyclosporine increases when combined with acitretin by inhibiting P-gp transporter. Hence, it shows that acitretin act as P-gp inhibitor.

Financial support and sponsorship

This work was self-funded. 


\section{Conflicts of interest}

There are no conflicts of interest.

\section{REFERENCES}

[1] FJ. Sharom, "The P-Glycoprotein Multidrug Transporter," Essays Biochem, Vol. 50, No. 1, pp 161-78, Sep. 2011.

[2] L. Saaby, B. A. Brodin, "Critical View on In vitro Analysis of Pglycoprotein (P-gp) Transport Kinetics," Journal of Pharmaceutical Science, vol. 106, no. 9, pp 2257-64, Sep 2017.

[3] RL. Linardi, CC. Natalini, "Multi-drug resistance (MDR1) gene and P-glycoprotein Influence on pharmacokinetic and pharmacodymanic of therapeutic drugs," Ciencia Rural, vol. 36, no. 1, pp. 336-41, Jan 2006.

[4] J T.Shikawa, H.Hirano, Y.Onishi, A.Sakurai, S.Tarui, "Functional Evaluation of ABCB 1 (P-Glycoprotein) Polymorphisms: HighSpeed Screening and Structure-Activity Relationship Analyses," Drug Metab Pharmacokinet, Vol.19, No.1, pp. 1-14, Feb 2004.

[5] ALA. Kuijpers, RJ. Van Dooren-Greebe, PCM. Van de Kerkhof, "Failure of Combination Therapy with Acitretin and Cyclosporin A in 3 Patients with Erythrodermic Psoriasis," Dermatolog, Vol. 194, No. 1, pp. 88-90, 1997.

[6] C. Chang, PM. Bahadduri, JE. Polli, PW. Swaan, S.Ekins, "Rapid Indentification of P-Glycoprotein Substrates and Inhibitors," Drug Metab Dispos, Vol. 34, 12, pp. 1976-84, Dec 2006.

[7] BL. Athukuri, P. Neerati, "Enhanced Oral Bioavailability of Domperidone with Piperine in Male Wistar Rats: Involvement of CYP3A1 and P-gp Inhibition," J Pharm Pharm Sci, vol. 20, pp. 2837, Jan 2017.

[8] Wahajuddin, KSR. Raju, SP. Singh, I. Taneja, "Investigation of the functional role of p-glycoprotein in limiting the oral bioavailability of lumefantrine," Antimicrob Agents chemother, vol. 8, no. 1, pp. 489-94,

Jan 2014.

[9] R. Jain, S. Duvvuri,V. Kansara, NK. Mandava, AK Mitra, "Intestinal absorption of noveldipeptide prodrugs of saquinavir in rats,"Int J Pharm, vol. 336, no. 2 , pp. 233-40, May 2007.

[10] S. Yigitaslan, K. Erol, C. Cengelli, "The effect of p-glycoprotein inhibition and activation on the absorption and serum levels of cyclosporine and tacrolimus in rats," Adv Clin Exp Med, vol. 25, no. 2, pp. 237-42, Mar-Apr 2016.

[11]D.Tsambaos, K.Bolsen, S.Georgiou, A.Kalofoutis, G.Goerz, "Effect of Oral Administration of Acitretin on Rat Liver Microsomal phospholipids, P-450 Content and Monooxygenase," Skin Pharmacol, vol. 7, pp. 320-3, Mar 1994.

[12]A. Parsa, R. Saadati, Z. Abbasian, S. Azad Aramaki, S. Dadashzadeh, "Enhanced Permeability of Etoposide across Everted Sacs of Rat Small Intestine by Vitamin E-TPGS," Iran J Pharm Res, vol. 12, pp. 37-46, Mar 2013.

\section{Author Contributions}

R.V conceived and designed the study. S.S.H.R conducted the experiments and drafted the manuscript under the guidance of R.V. Both authors have approved the final version of manuscript. 